\title{
CONFIGURAÇÕES ALTERNATIVAS PARA MAGNETÔMETROS "FLUXGATE" COM NÚCLEO AMORFO
}

\author{
Wanderlí Kabata e Ícaro Vitorello² \\ Recebido em 25 abril, 2007 / Aceito em 20 junho, 2007 \\ Received on April 25, 2007 / Accepted on June 20, 2007
}

\begin{abstract}
Fluxgate magnetometers are widely used in weak magnetic field measurements, mainly for purposes associated with geomagnetic observations, mineral prospecting and space research, because of their low power consumption, low noise level, large dynamic band and great sensitivity. Recent applications of soft magnetism in amorphous alloys have created a renewed stimulus to advance research in magnetic sensors, for deployment in tropical and sub tropical regions resembling Brazil. In the present study, ring cores made of amorphous ribbons are used to investigate the behavior of alternative fluxgate configurations to be introduced in the next generation of magnetometers to be developed at INPE for geomagnetic soundings. The results show the potential of temperature compensations in the fluxgate by the use of a modified feedback circuit, which provides, at the same time, the magnetic field feedback and the required magnetic field compensation due to temperature variations. The study of a direct-driven excitation provided by an amorphous tape-wrapped ring core provides support for a $2^{\text {nd }}$ harmonic magnetometer with sensitivity characteristics similar to those obtained with a conventional excitation, yet operated with less power consumption.
\end{abstract}

Keywords: magnetometers, fluxgate, direct excitation, geomagnetic soundings.

RESUMO. Magnetômetros fluxgates são amplamente usados em medidas do campo magnético de baixa intensidade, principalmente para finalidades associadas a observações geomagnéticas, prospecção mineral e pesquisa espacial, por causa do consumo pequeno, nível de ruído baixo, faixa dinâmica ampla e grande sensibilidade. Aplicações recentes de magnetismo mole, característico de ligas amorfas, têm criado novos estímulos para 0 avanço da pesquisa em sensores magnéticos para serem instalados em regiões tropicais e subtropicais como o Brasil. Neste estudo, núcleos feitos de fitas de ligas amorfas são usados na investigação de configurações alternativas a serem introduzidas na construção dos próximos magnetômetros construídos no INPE para sondagens geomagnéticas. Os resultados mostram 0 seu potencial para compensar variações de temperatura, com o uso de circuitos de realimentação modificados, que fornecem, ao mesmo tempo, a realimentação do campo magnético e a compensação do campo magnético pela variação da temperatura. 0 estudo da excitação direta fornecida pela fita de liga amorfa enrolada no núcleo confirma a ocorrência de características de sensibilidade magnética em um magnetômetro de segundo harmônico, similar às obtidas com a excitação convencional, porém com a vantagem do menor consumo de energia.

Palavras-chave: magnetômetro, fluxgate, excitação direta, sondagens geomagnéticas.

\footnotetext{
${ }^{1}$ INPE, Instituto Nacional de Pesquisas Espaciais, Divisão de Geofísica Espacial, Caixa Postal 515 - 12245-970 São José dos Campos, SP, Brasil. Tel.: (12) 3945-6749; Fax: (12) 39456810 - E-mail: wanderli@dge.inpe.br

2 INPE, Instituto Nacional de Pesquisas Espaciais, Divisão de Geofísica Espacial, Caixa Postal 515 - $12245-970$ São José dos Campos, SP, Brasil. Tel.: (12) 3945-6748; Fax: (12) 39456810 -E-mail: icaro@dge.inpe.br
} 


\section{INTRODUÇÃ̃o}

Os magnetômetros fluxgates têm sido desenvolvidos e utilizados há vários anos como sensores de campos magnéticos, principalmente nas aplicações que envolvam medidas do campo magnético terrestre, devido às suas condições ótimas de sensibilidade, ruído e particularmente por serem bastante robustos para aplicações de campo.

Com 0 avanço contínuo dos componentes eletrônicos, que conseguem a cada dia melhoras significativas em características como precisão, sensibilidade e estabilidade, há a necessidade de se desenvolver novos projetos, com novas configurações e abordagens diferentes, para se avançar no aprimoramento de sensores fluxgates, melhorando seu desempenho em sensibilidade e ruído. Vários grupos de pesquisa no mundo trabalham hoje em dia focando pontos onde ainda os fluxgates podem ser melhorados. Os pontos principais de atenção incluem a diminuição do ruído, a potência consumida, principalmente no circuito da excitação, e a miniaturização que tem como interesse principal a criação de "chips" fluxgates de precisão. Nesse trabalho, é realizado o desenvolvimento de novos circuitos eletrônicos e nova técnica de calibração de forma a diminuir os níveis de ruído dos fluxgates para levantamentos geofísicos de campo, porém mantendo a mesma sensibilidade da técnica convencional.

0 esforço atual no desenvolvimento de magnetômetros tipo fluxgate enquadra-se em nosso programa de desenvolvimento de equipamentos dedicados a levantamentos de dados geomagnéticos e telúricos no Brasil. Ele é alicerçado em experiências anteriores, que começaram com a construção de um magnetômetro fluxgate para estudos do eletrojato equatorial (Trivedi et al., 1995) seguido pela participação na construção de um magnetômetro fluxgate para o satélite de órbita polar (LEO) SACl-1, em colaboração com o grupo americano da IGPP/UCLA. A parte analógica daquele magnetômetro foi construída pela UCLA e a parte digital foi construída no laboratório de geomagnetismo do INPE (Trivedi et al., 2000). Um ano mais tarde, foi construído um magnetômetro fluxgate para 0 satélite de órbita equatorial SACl-2 (Kabata, 2000). Recentemente, foram confeccionados vinte magnetômetros fluxgate para sistemas de sondagens geomagnéticas profundas, que denominamos de GDSU - Geomagnetic Depth Sounding Units (Kabata et al., 2004).

Uma das grandes limitações pertinentes ao projeto de construção de magnetômetros fluxgate no Brasil, como o GDSU, é a obtenção do núcleo sensor cristalino, que é um material de venda controlada por causa de seu uso militar e difícil de ser adquirido no mercado internacional. As cooperações internacio- nais foram as formas utilizadas para as aquisições anteriores ao projeto GDSU.

Para os núcleos dos magnetômetros do projeto GDSU, outros novos materiais disponíveis no mercado foram testados. Finalmente, materiais amorfos foram selecionados e utilizados com sucesso em novas configurações que permitiram diminuir o nível de ruído. Atualmente, entre as configurações testadas está aquela em que não se utiliza o clássico enrolamento primário para gerar o campo magnético necessário para saturar o núcleo, sendo que a saturação é conseguida aplicando-se uma corrente elétrica diretamente no material magnético do núcleo, assunto a ser detaIhado no texto abaixo.

\section{PRINCÍPIO DE FUNCIONAMENTO}

0 aparecimento de uma força eletromotriz induzida, gerada pela variação do fluxo magnético, é largamente usada na detecção de campos magnéticos, em magnetômetros tipo fluxgate.

Colocando-se uma bobina com um núcleo ferromagnético imerso num campo externo, as linhas de campo se concentram ao passarem pelo núcleo devido a sua alta permeabilidade magnética $(\mu)$. No entanto, nenhuma força eletromotriz é gerada, devido ao fluxo magnético ser constante. Nota-se, portanto, a necessidade de se ter variações nesse fluxo para se conseguir alguma medida.

A variação do fluxo magnético pode ser espontânea, se 0 campo que se queira medir for variável no tempo, ou provocada, onde pode ser feita a variação do fluxo indiretamente, ou pela variação da permeabilidade magnética do meio, ou pela variação da área por onde atravessam as linhas de campo. Pode-se, por exemplo, rodar uma espira num campo estático que se queira medir, onde a tensão induzida será proporcional ao campo, à área da espira e à freqüência de rotação. Esse tipo de magnetômetro é conhecido como magnetômetro rotativo de indução, porém não é usado, hoje em dia, para medidas do campo geomagnético.

Outra possibilidade de termos variações do fluxo magnético consiste em variar a permeabilidade do meio que é circundado pela bobina (núcleo), em outras palavras, variar a permeabilidade do núcleo da bobina.

Os magnetômetros de núcleo saturado, ou "fluxgate magnetometer", são dispositivos que medem a magnitude e direção de campos magnéticos. São capazes de medir campos estáticos ou alternados e tem seu funcionamento baseado nas propriedades de saturação de ligas magnéticas moles, que possibilitam 0 chaveamento do fluxo magnético, devido à variação da permeabilidade magnética do material usado como núcleo. Se o núcleo tiver alta permeabilidade, o campo externo será "canalizado" para dentro 
dele e 0 fluxo que atravessa a espira será muito grande (Fig. 1a). Se por alguma razão a permeabilidade do meio diminuir repentinamente, o campo deixará de se concentrar dentro da espira e o fluxo devido ao campo externo diminuirá (Fig. 1b). Nesse instante, a variação do fluxo provoca uma tensão induzida proporcional ao campo externo a que se quer medir.

0 controle da permeabilidade do material é possível através de um campo magnético adicional, denominado campo magnético de excitação, gerado a partir de um enrolamento chamado enrolamento de excitação ou simplesmente enrolamento primário. Acontece que, quando não há corrente passando no primário, a permeabilidade do núcleo é a própria permeabilidade do material, que pode atingir valores altíssimos para materiais ferromagnéticos.

Se uma corrente suficientemente alta é aplicada no primário, de tal forma a gerar um campo magnético $H$ maior que 0 campo magnético de saturação do material $H_{S}$, então a permeabilidade do núcleo decresce para valores próximos ao da permeabilidade do vácuo, como se o núcleo tivesse desaparecido do interior da bobina.

Envolvendo todo o conjunto (bobina de excitação e núcleo), existe um enrolamento denominado bobina detectora ou simplesmente enrolamento secundário. Ela é a responsável pela detecção da variação do fluxo magnético. Cada vez que o núcleo passa da condição de saturado para não saturado, ou vice versa, as linhas de campo magnético que cortam as espiras da bobina detectora induzem pulsos. Desta forma, dois pulsos são gerados a cada ciclo, e são amostrados com um sinal de freqüência duas vezes maior que a frequiência de excitação. A amplitude e fase de cada um dos harmônicos do sinal presentes no secundário são proporcionais à magnitude e à polaridade do campo magnético externo presente ao longo do eixo do enrolamento secundário.

\section{DESENVOLVIMENTO DE NOVAS CONFIGURAÇÕES}

A Figura 2 mostra 0 diagrama de blocos de um magnetômetro fluxgate de precisão contendo o modo convencional de corrigir a fase do sinal magnético e de amostrá-lo sincronamente. Como é necessário um sinal no detector síncrono que esteja sincronizado com a corrente de excitação, utiliza-se um sinal de referência com uma freqüência duas vezes maior que a da excitação, conhecida como referência $2 f$, a qual tem a finalidade de temporizar a amostragem no detector síncrono.

0 ajuste de fase, importante para aumentar a sensibilidade e diminuir o ruído, é realizado na forma de onda do sinal magnético medido, antes deste chegar ao circuito de detecção síncrona.
A experiência acumulada nos trabalhos anteriores (Kabata et al., 2003, 2004) e o desenvolvimento e utilização de vários circuitos de fluxgates mostraram que a inserção de muitos circuitos em série na linha principal do magnetômetro (linha que vai desde a bobina de detecção até 0 circuito integrador, mostrada nos diagramas de blocos) torna 0 magnetômetro muito mais sensivel às variações de temperatura. Efeitos da variação da temperatura, principalmente nos amplificadores operacionais, muitas vezes produzem ruídos que podem ser maiores do que 0 sinal magnético medido.

0 circuito tem melhor desempenho em relação à estabilidade de fase quando se retira 0 ajuste de fase do ramo principal e coloca-o no circuito digital, atrasando ou adiantando o sinal de referência de forma a compensar as mudanças de fase do sinal magnético. Ou seja, ao invés de fazer 0 ajuste da fase em cima do sinal magnético, faz-se o ajuste em cima do sinal de referência (Fig. 3). 0 circuito fica mais estável, pois 0 circuito digital utilizado para adiantar ou atrasar o sinal de amostragem no detector síncrono sofre menos com as variações de temperatura do que um amplificador operacional em série na linha principal do magnetômetro.

No entanto, mesmo que haja variações no circuito do relógio (gerador de "clock"), como o circuito de compensação de fase é colocado antes de ser referenciado pela excitação e pela amostragem síncrona, qualquer variação afetará ambas de igual forma e, portanto não terá efeito de avanço ou atraso de fase.

Quando acontece alguma variação de tempo no gerador de "clock" devido às variações de temperatura, o mesmo acontece na excitação e também no circuito de correção de fase, tornandoos sempre sincronizados.

\section{Compensação de temperatura}

A dependência do sinal magnético medido com a variação da temperatura nos magnetômetros fluxgates é um grande problema a ser resolvido.

Esse fato ocorre principalmente por duas razões. A primeira é devido à variação da resistividade do enrolamento do fio de cobre que envolve a bobina. Essa variação resistiva provoca variações na corrente e, portanto, no campo de realimentação do magnetômetro $\left(B_{R}\right)$, campo responsável por deixar o ambiente do núcleo em campo magnético quasi-nulo. A segunda razão é devida à variação do tamanho da bobina associada às variações da temperatura, que também provoca variações no campo de realimentação $B_{R}$. Essas variações do campo de realimentação, tanto no primeiro quanto no segundo caso, aparecem na saída do 


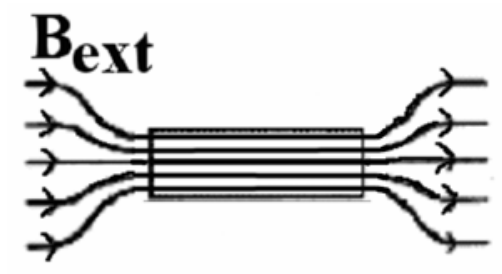

a) Núcleo com alto $\mu$.

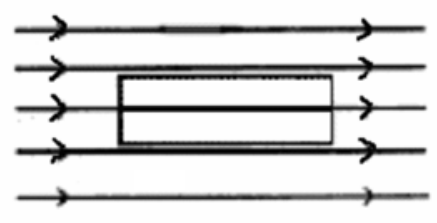

b) Núcleo com baixo $\mu$.

Figura 1 - Variações do fluxo magnético.

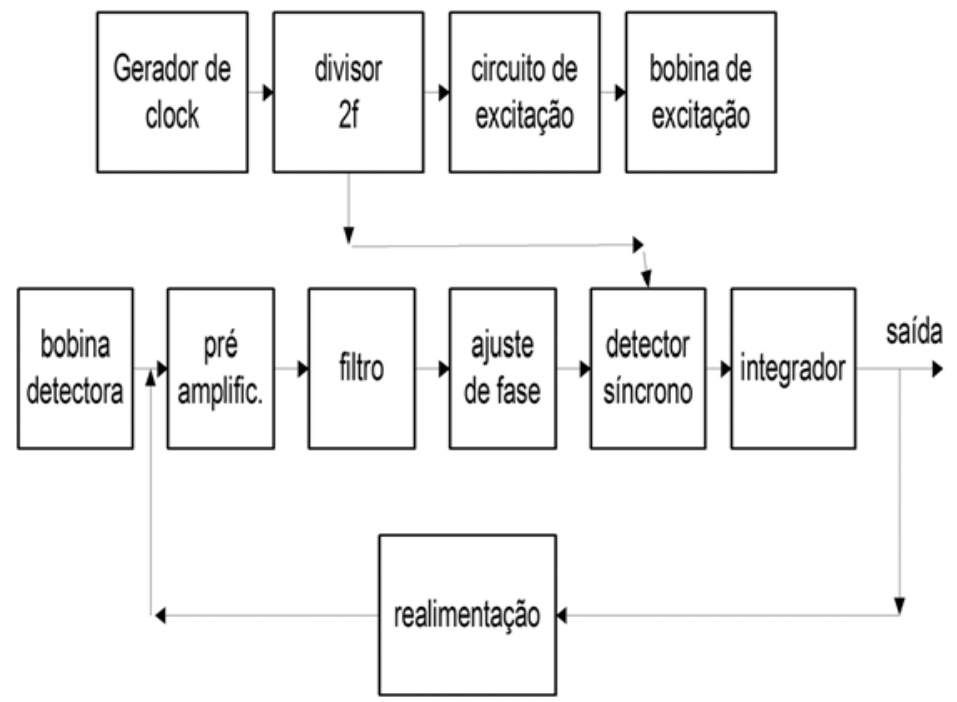

Figura 2 - Diagrama de blocos de um fluxgate convencional.

magnetômetro como se fossem variações do campo magnético medido, provocando erros nas medidas.

Desde o experimento SACI2 (Kabata, 2000) temos utilizado um amplificador conversor tensão corrente que gera um campo de realimentação para manter o núcleo em campo magnético próximo à zero.

A técnica de medir a temperatura com 0 próprio enrolamento do magnetômetro (Primdahl, 1970; Acuña, 1978; Fieberg \& Kuhnke, 1994), e ao mesmo tempo podê-la corrigir, é mais facilmente aplicada utilizando-se de magnetômetros com o mesmo enrolamento para a detecção e para a realimentação. Nesse caso, mede-se a variação da resistência do fio do enrolamento e utiliza-se de um circuito que corrija a variação que ocorre no próprio enrolamento e que dê maior estabilidade ao sensor. Para esse experimento, foi desenvolvido um magnetômetro com núcleo de fita vitrometálica amorfa tendo apenas dois enrolamentos (Kabata et al., 2004)

0 campo de realimentação $B_{R}$, que flui através do enrola- mento secundário de modo a anular o campo medido, depende também do tamanho da bobina. Como a temperatura varia, há variações incorretas no campo de compensação. Essas variações são causadas não pela mudança real no campo medido, e sim por uma mudança na temperatura do sensor, por isso, deve ser evitada.

0 campo magnético dentro de um solenóide depende basicamente da corrente $I$, do número de espiras $N$, do comprimento médio $l$, e do raio $R$.

$$
B_{R}=\frac{\mu_{0} N I}{\sqrt{4 R^{2}+I^{2}}} .
$$

Esse campo quando realimentado sofre flutuações devido às variações do tamanho e da resistência do solenóide. A forma de corrigir essas variações foi concatenada através de um circuito que consegue criar resistências negativas, compensando as variações que ocorrem no solenóide devido à mudança na temperatura. 


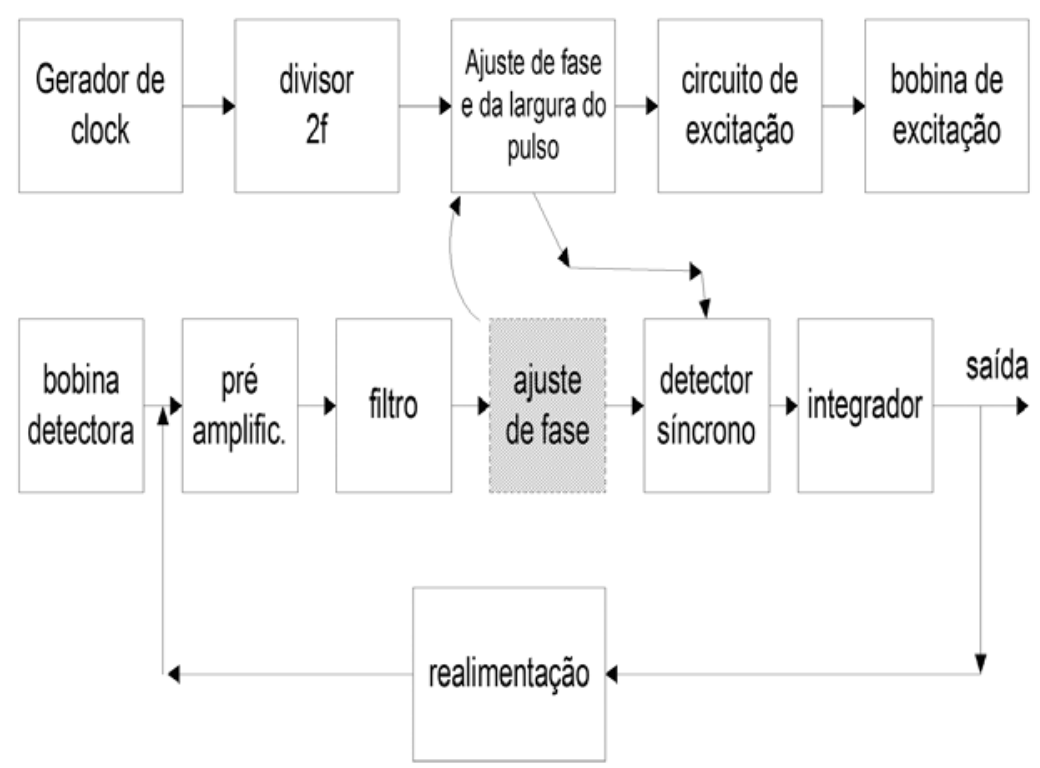

Figura 3 - Diagrama de blocos do fluxgate mostrando a mudança do local do circuito de correção de fase.

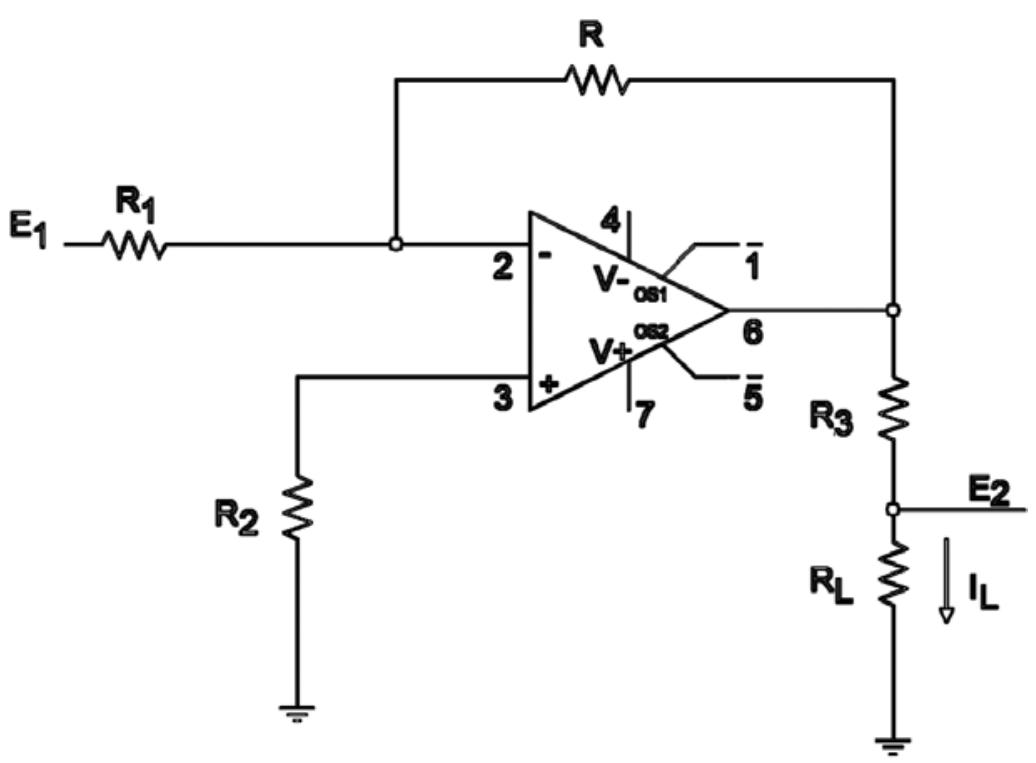

Figura 4 - Circuito conversor tensão corrente.

Como já estava sendo usado um circuito conversor tensão corrente para a realimentação do fluxgate (Fig. 4), esse circuito foi modificado para também ter a função de minimizar os efeitos da variação da temperatura no enrolamento secundário (bobina sensora). Desta maneira, atua contrariamente, corrigindo os valores do campo devido às variações da temperatura, além de fazer 0 trabalho normal de realimentação negativa do campo, para que o núcleo trabalhe em campo magnético próximo a zero.
Portanto, esse novo circuito utiliza 0 enrolamento secundário como sensor campo magnético e de temperatura, e atua normalmente como realimentação negativa do campo medido, além de corrigir as flutuações devido às variações de temperatura, tornando o magnetômetro bastante estável.

A natureza da dependência do sensor fluxgate com a temperatura ainda não é completamente compreendida, no entanto, já é reconhecido que 0 efeito da temperatura é proporcional ao 
campo medido. Acredita-se que a maior fonte de desvios seja devido a um campo adicional causado pela corrente de realimentação. As mudanças nas tensões de "offsel" do sensor em campo nulo, ou na sensibilidade, devem causar flutuações, mas estima-se que essas contribuições são insignificantes, já que 0 sensor não mostra dependência de temperatura em campos com valores próximos à zero (campo nulo).

0 circuito utilizado para gerar o campo de realimentação e de compensação devido à variação da temperatura é o indicado na Figura 5. A tensão $E_{2}$ é apenas um ponto de leitura da tensão em cima do enrolamento $R_{L}$ da bobina.

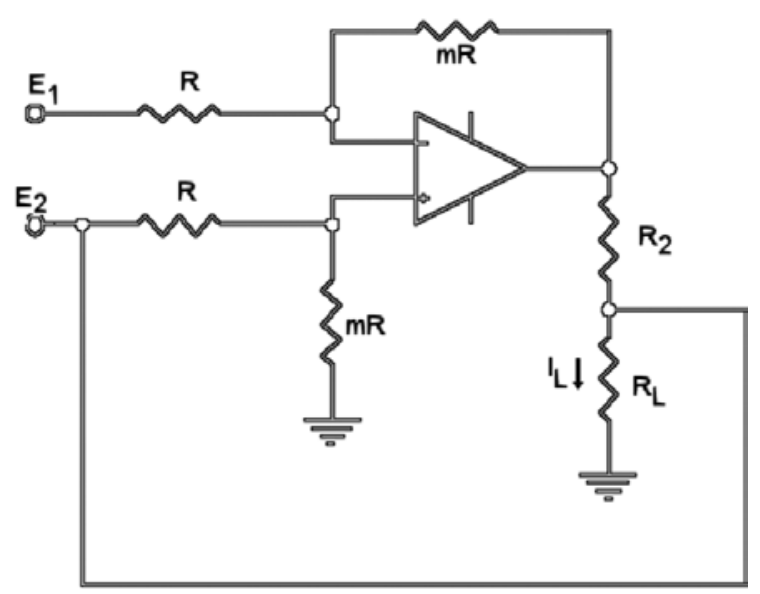

Figura 5 - Novo circuito de realimentação.

Como praticamente não circula corrente pelo pino positivo $(+)$ do amplificador operacional, podemos encontrar $E_{2}$ como no circuito da Figura 6:

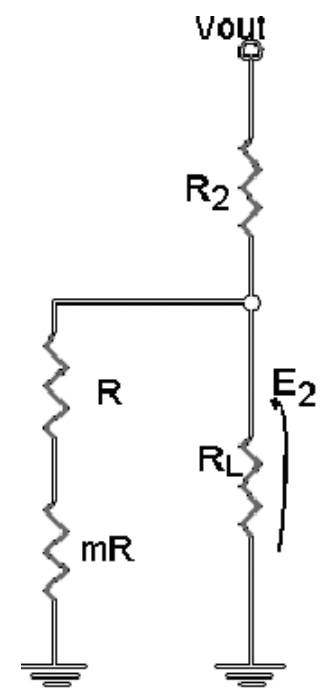

Figura 6 - Circuito equivalente da tensão $E_{2}$

$$
E_{2}=\frac{V_{\text {out }}\left[R_{L} / /(R+m R)\right]}{\left[R_{2}+R_{L} / /(R+m R)\right]}
$$

onde

$$
R_{L} / /(R+m R)=\frac{R_{L}(R+m R)}{R_{L}+(R+m R)}
$$

0 circuito utilizado é basicamente um amplificador diferencial modificado de forma a utilizar a tensão em cima do enrolamento de detecção somando-a com o sinal vindo do integrador. 0 resultado é observado na tensão de saída que realimenta a bobina. Esse amplificador diferencial, modificado quando conectado ao enrolamento de detecção, de resistência $R_{L}$ funciona como um circuito de impedância negativa. Esse processo provoca um aumento da corrente $I_{L}$ com 0 aumento da resistência $R_{L}$, e uma diminuição da corrente $I_{L}$ com a diminuição de $R_{L}$, funcionando como uma reação contrária ao aumento ou à diminuição da resistência do fio de cobre, os quais são provocados pelo aumento ou diminuição da temperatura. 0 circuito funciona como se houvesse criado uma resistência negativa, que anula a variação da resistência do conjunto (enrolamento, cabo) devido às variações da temperatura o que, por conseguinte, impede que o campo de compensação varie. Para estabilizar o circuito na faixa de temperatura desejada, deve-se definir os valores de $R, m R$ e $R_{2}$ do circuito da Figura 5, montando o magnetômetro inclusive com os cabos de operação, pois o circuito operará sobre as variações de todo o conjunto, melhorando a estabilidade térmica. Como o campo de realimentação $\left(B_{R}\right)$ é proporcional à corrente de realimentação e ao comprimento da bobina (coil), os valores dos resistores do circuito que compensam as variações de temperatura são encontrados diferenciando $B_{R}$ em relação à temperatura (Acuña, 1978). Na prática, é feita a variação da temperatura de todo o conjunto e escolhido os valores dos resistores que melhor se ajustam. Com isso, também já está sendo considerada a mudança no valor do campo de compensação devido à mudança no tamanho da bobina.

\section{Simulação dos circuitos de compensação e cálculo da variação do campo de realimentação}

A corrente de realimentação fluindo através da bobina de detecção gera um campo de realimentação $B_{R}$ que é dependente da variação do tamanho da bobina e da variação da resistividade do cobre que aumenta ou diminui a resistência do enrolamento. 0 material utilizado para suporte da bobina é um policarbonato com coeficiente de temperatura de $50.10^{-6} / \mathrm{K}$. 0 coeficiente de temperatura do cobre é 4,33.10-3/K. 
Simulando o circuito conversor tensão corrente (foi utilizado o programa PSPICE versão estudante "free evaluation"), geralmente utilizada para gerar o campo de realimentação, teremos as seguintes respostas:

Tabela 1 - Valores da corrente $I_{L}$ devido às variações na resistência $R_{L}$ para diferentes valores de temperatura para 0 circuito da Figura 4.

\begin{tabular}{|c|c|c|c|}
\hline$T\left[{ }^{\circ} \mathrm{C}\right]$ & $R_{L}[\Omega]$ & $I_{L}[m A]$ & $V_{L}[\mathrm{~V}]$ \\
\hline 20 & 75,0 & 1,79453 & $-134,59$ \\
\hline 37 & 80,0 & 1,79163 & $-143,33$ \\
\hline 40 & 81,5 & 1,79079 & $-145,95$ \\
\hline 51 & 85,0 & 1,78871 & $-152,04$ \\
\hline
\end{tabular}

Notamos que à medida que a resistência $R_{L}$ aumenta, há uma diminuição da corrente $I_{L}$ que flui sobre $R_{L}$, gerando 0 campo de realimentação $B_{R}$.

Se considerarmos apenas a variação de tamanho, (sem variação da resistência $R_{L}$ e, portanto com $I_{L}$ constante) num solenóide de dimensões $L=4 \mathrm{~cm}$ e $R=1 \mathrm{~cm}$ com coeficiente de temperatura do suporte de $5010^{-6} / \mathrm{K}$ para uma bobina com 600 espiras e uma corrente de 1,7953 mA fluindo no enrolamento, teremos os seguintes resultados no caso de uma temperatura inicial de $20^{\circ} \mathrm{C}$ e final de $40^{\circ} \mathrm{C}$ :

$$
\begin{aligned}
B_{1}= & \frac{4 \pi \cdot 10^{-7} \times 600 \times 1,7953.10^{-3}}{\sqrt{4.0,01^{2}+0,04^{2}}} \\
= & 30267,96 n T \\
B_{2}= & \frac{4 \pi \cdot 10^{-7} \times 600 \times 1,7953.10^{-3}}{\sqrt{4.0,01001^{2}+0,04004^{2}}} \\
= & 30.237,96 n T \\
& B_{1}-B_{2}=30,24 n T
\end{aligned}
$$

sendo:

$B_{1}=$ Campo de realimentação a $20^{\circ} \mathrm{C}$ (condição inicial).

$B_{2}=$ Campo de realimentação a $40^{\circ} \mathrm{C}$ considerando apenas a variação no tamanho da bobina, ou seja, sem variação na corrente de realimentação.

Notamos que mesmo utilizando materiais bastante estáveis em relação à temperatura, como é o caso do policarbonato utilizado $\left(\alpha=5010^{-6} /{ }^{\circ} \mathrm{C}\right)$, há grandes variações na medida do campo devido à variação no tamanho da bobina.

Considerando, agora, apenas a variação da resistência $R_{L}$ do enrolamento de cobre, tomando os valores de corrente da Tabela 1 para os valores de temperatura de $20^{\circ} \mathrm{C}$ e $40^{\circ} \mathrm{C}$, podemos calcular:

$$
\begin{gathered}
B_{3}=\frac{4 \pi \cdot 10^{-7} \times 600 \times 1,79079.10^{-3}}{\sqrt{4.0,01^{2}+0,04^{2}}} \\
=30191,9 n T \\
B_{1}-B_{3}=76,06 n T
\end{gathered}
$$

Sendo $B_{3}$ o campo de realimentação considerando que não houve alteração no tamanho da bobina, apenas na resistência do enrolamento e, portanto, na corrente de realimentação.

Refazendo os testes considerando as variações do campo de realimentação devido aos dois fatores conjuntamente, ou seja, com variações no tamanho da bobina e na resistência do fio, podemos calcular:

$$
\begin{gathered}
B_{4}=\frac{4 \pi \cdot 10^{-7} \times 600 \times 1,79079.10^{-3}}{\sqrt{4.0,01001^{2}+0,04004^{2}}} \\
=30.161,77 n T \\
B_{1}-B_{4}=106,19 n T
\end{gathered}
$$

Verificamos que existe uma grande influência da temperatura nos valores medidos devido às mudanças nos valores do campo de realimentação, ou seja, uma diferença de 106 nT no campo de realimentação que influenciará diretamente o valor do campo medido, como se tivesse havido mudança no campo externo medido e não no campo de realimentação.

Utilizando o circuito de realimentação com compensação de temperatura (Fig. 5) e simulando as condições de operação, teremos os seguintes resultados:

Tabela 2 - Valores da corrente $I_{L}$ devido às variações na resistência $R_{L}$ para diferentes valores de temperatura para 0 circuito da Figura 5.

\begin{tabular}{|c|c|c|c|}
\hline$T\left[{ }^{\circ} \mathrm{C}\right]$ & $R_{L}[\Omega]$ & $I_{L}[\mathrm{~mA}]$ & $V_{L}[\mathrm{~V}]$ \\
\hline 20 & 75,0 & 1,8600 & $-139,50$ \\
\hline 37 & 80,0 & 1,861375 & $-148,91$ \\
\hline 40 & 81,5 & 1,861718 & $-151,73$ \\
\hline 51 & 85,0 & 1,862706 & $-158,33$ \\
\hline
\end{tabular}

Calculando os campos de realimentação para as temperaturas de $20^{\circ} \mathrm{C} \mathrm{e} 40^{\circ} \mathrm{C}$ teremos, para o pior caso, ou seja, com a variação do tamanho e também com a variação da resistividade 
os seguintes valores:

$$
\begin{aligned}
& B_{5}= \frac{4 \pi \cdot 10^{-7} \times 600 \times 1,86000.10^{-3}}{\sqrt{4.0,01^{2}+0,04^{2}}} \\
&= 31358,77 n T \\
& B_{6}= \frac{4 \pi \cdot 10^{-7} \times 600 \times 1,861718.10^{-3}}{\sqrt{4.0,01001^{2}+0,04004^{2}}} \\
&= 31.356,38 n T \\
& B_{5}-B_{6}=2,39 n T
\end{aligned}
$$

onde:

$B_{5}=$ Campo de realimentação (condição inicial).

$B_{6}=$ Campo de realimentação levando em consideração as variações de tamanho e resistência do enrolamento da bobina.

Esses resultados demonstram a possibilidade de se corrigir não somente a influência da variação da resistência elétrica do cobre como também a influência da variação do tamanho da bobina para as faixas de variações de temperatura desejadas. Tem-se a vantagem de utilizar o mesmo amplificador do circuito que gera o campo de realimentação, ou seja, não há necessidade de se inserir circuitos integrados adicionais, o que representaria aumento de consumo.

Medidas realizadas com 0 sensor colocado em câmara de blindagem magnética, com um campo de 25.000 nT aplicado em cada eixo separadamente, apresentaram variações menores que $1 \mathrm{nT} /{ }^{\circ} \mathrm{C}$, na faixa de $25^{\circ} \mathrm{C}$ a $35^{\circ} \mathrm{C}$.

\section{Experimento magnetômetro fluxgate amorfo com excitação direta}

Hoje em dia, as principais tendências para os estudos dos sensores magnéticos tipo "fluxgate" referem-se à miniaturização, ao aumento da sensibilidade e à redução do nível de ruído magnético do sensor. A redução de tamanho tem como conseqüência a perda de sensibilidade, daí a necessidade de estudos para o aumento de sensibilidade e redução do ruído ganha maior importância. Estudos abordando redução de consumo sempre estão em alta demanda. Assim, S.C. Tang (Tang et al., 2004) ganhou o prêmio de melhor trabalho apresentado no congresso da Eurosensors em 2002, abordando a utilização em série de indutores saturáveis na excitação de magnetômetros fluxgates, podendo, dessa forma, excitar os magnetômetos com tensões mais baixas que as convencionais. Ripka (2003) abordou a técnica de ressonância em série como alternativa para excitar magnetômetros fluxgates com um simples chaveamento de baixa tensão. Ioan et al.
(2002) utilizaram fitas amorfas numa configuração VacquierFoster (haste dupla), utilizando-se de dupla excitação, via enrolamento primário e via direta, como forma de se obter um magnetômetro fluxgate de alta resolução.

Complementamos as configurações alternativas, a seguir, com um estudo baseado na aplicação da corrente de excitação diretamente na fita que forma o núcleo em anel, para se obter as necessárias variações de permeabilidade do núcleo de um fluxgate.

\section{RESULTADOS COM A EXCITAÇÃO DIRETA}

Em um magnetômetro fluxgate convencional, o circuito de excitação, responsável por proporcionar as variações da permeabilidade magnética do núcleo, é realizada com uma bobina com várias espiras enroladas em torno do núcleo sensor, de tal forma a criar um campo magnético alternado que leva periodicamente 0 núcleo ao estado de saturação.

Este estudo apresenta um novo fluxgate em anel enrolado com uma fita dupla do material amorfo vitrovac, tratada termicamente para alívio das tensões e fixação do eixo de anisotropia magnética. A fita dupla, na realidade, é a mesma fita utilizada para a confecção dos núcleos do projeto GDSU, no entanto ela foi dobrada antes de ser enrolada no suporte do núcleo, conforme mostra a Figura 7. Dobrando a fita dessa forma, o ruído do efeito transformador que seria causado pela corrente que passa pela fita é anulado, pois a mesma corrente passa em um sentido na fita em cima e em sentido contrário em baixo.

A fita, de $20 \mu \mathrm{m}$ de espessura, foi colocada de forma a não ter espaço entre a parte de cima e a de baixo, ficando a dupla fita com aparência de apenas uma, porém com o dobro da espessura, e depois foi enrolada num suporte em forma de anel. A curvatura da fita, de aproximadamente $1 \mathrm{~mm}$, é escondida através de um rasgo num plano perpendicular ao plano que será enrolada a fita, de forma a permitir que a espessura dupla da fita esteja em toda a circunferência do núcleo.

Ao ser ajustada cuidadosamente uma face em cima da outra, o efeito externo da corrente passando pelo lado de cima é anulada pela corrente retornando pelo lado de baixo. Com esse artifício, espera-se uma menor indução da corrente de excitação na bobina de detecção pelo efeito transformador. Depois de dobrada, ela é enrolada Iongitudinalmente no suporte. Foram enroladas 10 voltas da fita, perfazendo um total de 20 camadas de espessura de material amorfo no núcleo. Nas extremidades da fita foram soldados terminais de cobre e conectados ao circuito gerador de corrente. 0 conjunto todo foi colocado dentro de um suporte, for- 


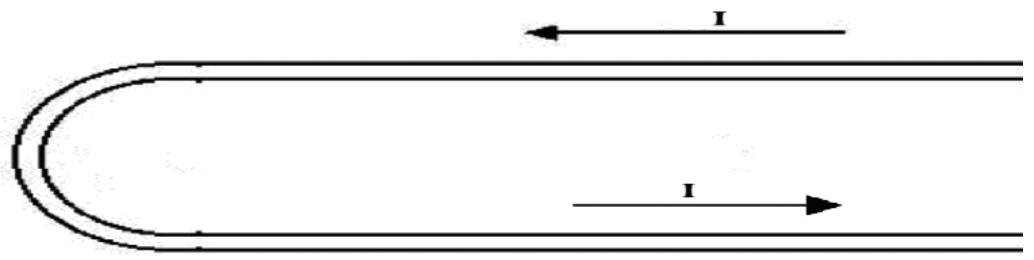

Figura 7 - Forma para dobrar a fita do núcleo.

mando o núcleo. Sobre esse núcleo, foi enrolada uma bobina com 550 espiras, por onde 0 sinal magnético é detectado. Esse sinal, após ser filtrado, amostrado sincronamente e integrado, fornece 0 sinal de tensão de saída equivalente ao campo magnético externo.

Para a excitação direta, foi utilizado um gerador de corrente de excitação de 15,6 kHz com amplitudes variáveis de corrente, aplicado diretamente num circuito ressonante serial, (Ripka, 2003), conforme mostra a Figura 8.

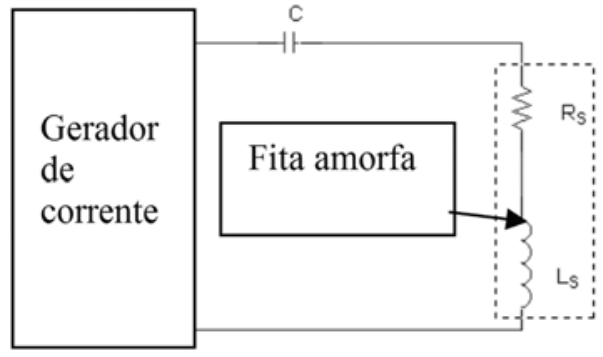

Figura 8 - Esquema para excitação direta.

Na Figura 8, os símbolos representam:

$L_{s}=$ Indutância do núcleo para 0 estado saturado; ou

$L_{n}=$ Indutância do núcleo para o estado não saturado;

$C=$ Capacitor série.

0 cálculo dos parâmetros de excitação leva em conta os estado de saturação e não saturação da fita, obtendo-se os valores a partir da seguinte formulação:

$$
\omega_{n}=\frac{1}{\sqrt{L_{n} C}}, \quad \omega_{s}=\frac{1}{\sqrt{L_{s} C}}, \quad \Delta=\pi \sqrt{L C}
$$

onde $\Delta=$ Largura do pulso de corrente.

0 capacitor $C$ serve também para eliminar a componente $D C$ da corrente de excitação, o que poderia causar graves erros nos valores da tensão de "offset", devido à assimetria na forma de onda de excitação.

A forma de onda da corrente de excitação, com sua aplicação direta nos terminais da fita, apresentou uma forma de onda muito parecida com aquela que se obtém no modo convencional (Fig. 9), tendo pulsos finos de corrente e com um valor de pico mais baixo do que na excitação convencional via bobina de excitação, fato importante para diminuir o consumo de energia do fluxgate.

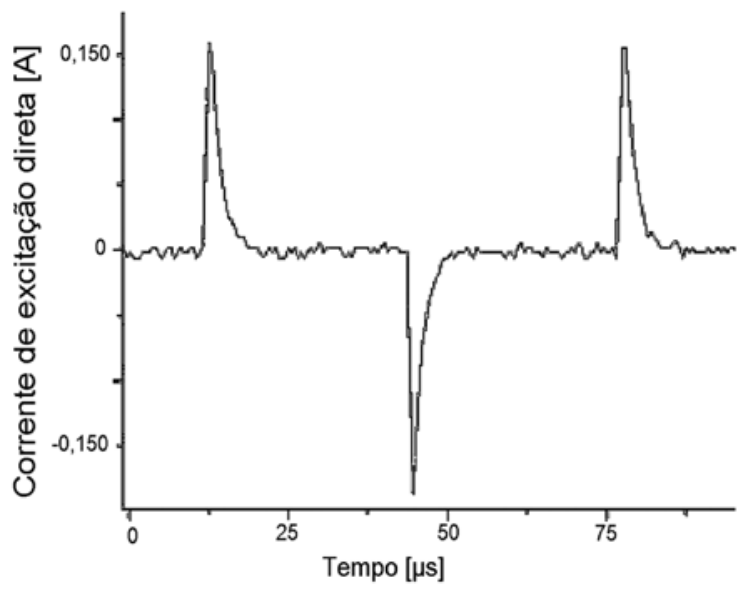

Figura 9 - Forma de onda da excitação direta capturada da tela do osciloscópio.

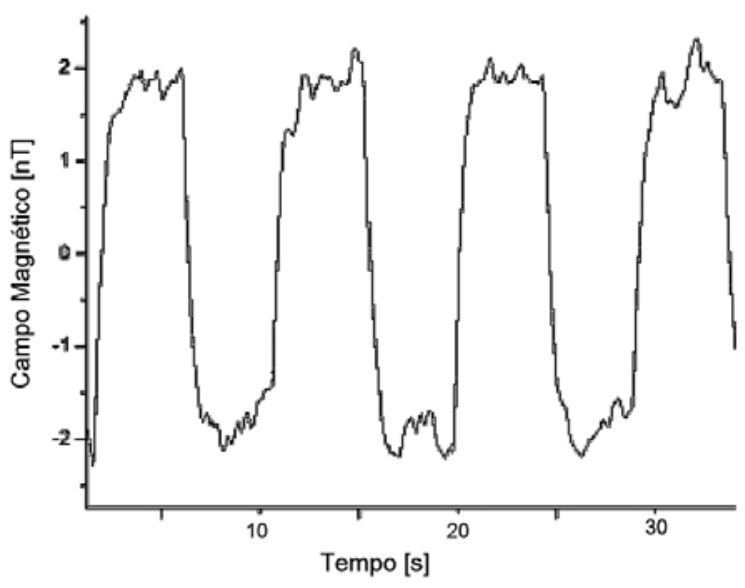

Figura 10 - Sinal magnético para $\pm 2 n T$.

Colocando o magnetômetro fluxgate dentro de uma câmara de blindagem magnética de três camadas de mu-metal (liga de $\mathrm{Ni}$, $\mathrm{Fe}, \mathrm{Cu}, \mathrm{Mo}$ com alta permeabilidade) e aplicando-se um campo magnético pulsante entre os valores de +2nT e -2nT obtém-se uma resposta mais satisfatória do funcionamento geral do fluxgate (Fig. 10). Este resultado foi obtido apesar das condições desfavoráveis por ocasião dos testes no laboratório devido às grandes variações magnéticas provocadas por movimentação de móveis, 


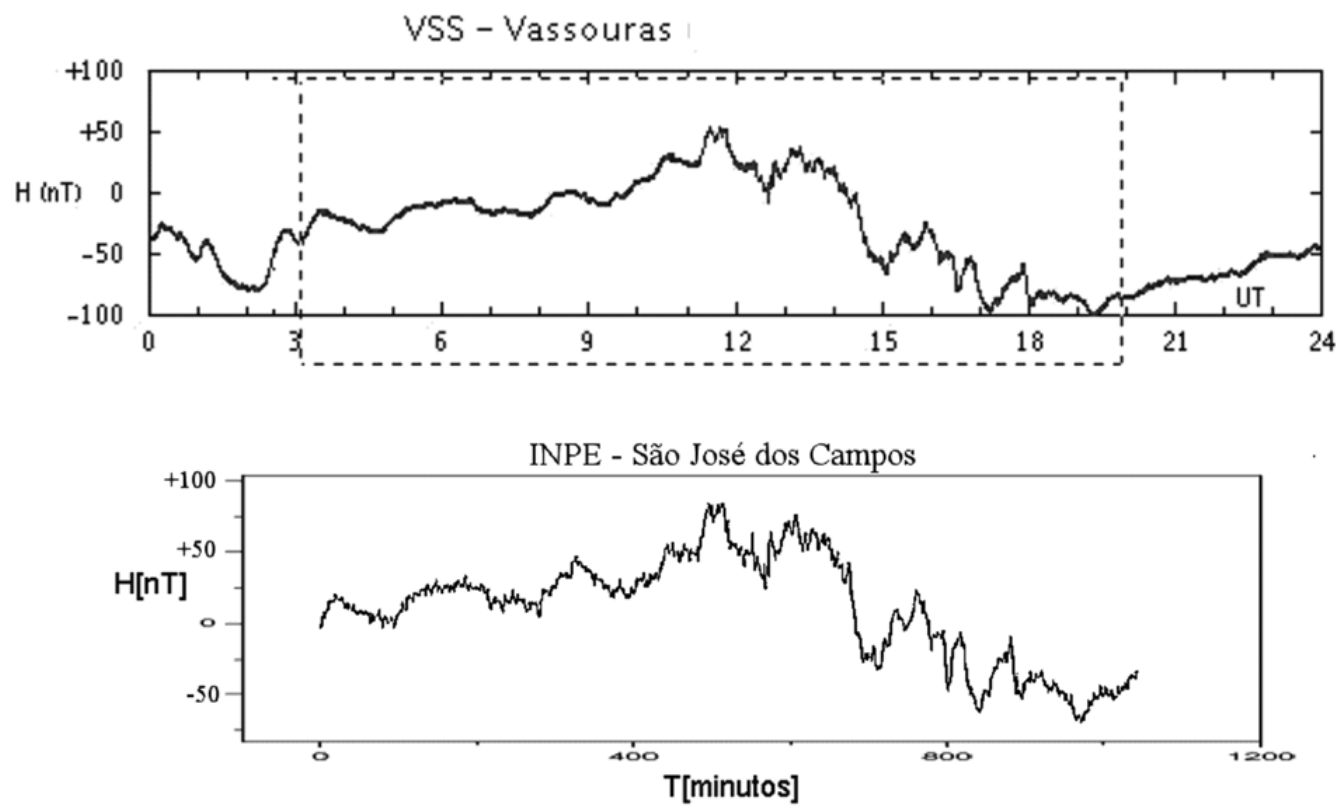

Figura 11 - Variações magnéticas do dia 08 de maio de 2005, obtidas em Vassouras (RJ) e em São José dos Campos (SP).

veículos passando a menos de $10 \mathrm{~m}$ do local, e outros ruídos eletromagnéticos aleatórios.

Medidas das variações do campo magnético terrestre, mesmo em condições ruins, como o do laboratório, comprovam que este magnetômetro responde bem às variações do campo quando comparado com outras determinações simultâneas, no caso, comparando os dados obtidos no laboratório localizado em São José dos Campos (SP) com os do Observatório Magnético de Vassouras (RJ).

Na Figura 11, 0 gráfico do Observatório de Vassouras (gráfico acima) mostra as variações magnéticas do dia 08 de maio de 2005. 0 eixo das abscissas mostra o tempo em horas para um dia completo. Os dados tomados no laboratório (gráfico abaixo) foram tomados das 3:00 às 20:00, com 0 eixo das abscissas mostrando o tempo em minutos. Para facilitar a comparação dos dados, foi tracejado o período de coleta simultânea dos dados no gráfico do Observatório de Vassouras.

As variações medidas em laboratório com o magnetômetro de excitação direta tiveram comportamentos coerentes com as variações diurnas observados em Vassouras. Adicionalmente, 0 magnetômetro ainda mostrou sensibilidade nos testes quando excitado com variações de campos magnéticos conhecidos. Portanto, é possível construir bons magnetômetros fluxgate com esta técnica, a qual permite simplificar a construção, porque não necessita do enrolamento primário, e também diminuir o consumo, pois utiliza uma corrente de excitação direta de menor intensidade para saturar a fita, em comparação com o método convencional.

\section{CONCLUSÃO}

0 trabalho com magnetômetros fluxgate, realizado nos últimos anos, mostra a importância do investimento em pesquisa de desenvolvimento em tecnologia para a sustentação e criação de novos projetos de pesquisa utilizando-se desse desenvolvimento tecnológico.

Aplicando-se as novas técnicas aqui apresentadas, esperase obter equipamentos com maior estabilidade em relação às variações da temperatura e menores ruídos. Em relação ao estudo para o desenvolvimento de um magnetômetro fluxgate com excitação direta, o estudo realizado mostrou que este magnetômetro opera nas mesmas condições de um magnetômetro convencional. Apresenta uma excelente resposta ao sinal geomagnético e com a vantagem do menor consumo, devido ao valor da corrente aplicada diretamente na fita para conseguir que a profunda saturação do núcleo seja menor para este tipo de excitação. Seu circuito é mais simples, já que não existe a bobina de excitação, o que poderia facilitar sua integração em pastilhas para a obtenção de circuitos integrados de magnetômetros fluxgate de alto desempenho. No entanto, é necessário construir protótipos a serem testados por um longo período no campo, para uma melhor avaliação. 


\section{REFERÊNCIAS}

ACUÑA MH. 1978. The MAGSAT Vector Magnetometer - A Precision fluxgate Magnetometer for the measurement of the Geomagnetic Field, NASA, Technical Memorandum 79656. 20 pp.

FIEBERG F \& KUHNKE F. 1994. The increase of the magnetic field in the KTB pilot and main drillhole. Scientific Drilling 4: 139-145.

IOAN C, CHIRIAC H, DIACONU ED, MOLDOVANU A, MOLDOVANU E \& MALCOVEI C. 2002. High-resolution fluxgate sensing elements using Co68,25Fe4,5Si12,25B15 amorphous material. Journal of Optoelectronics and Advanced Materials, 4(2): 319-324.

KABATA W. 2000. Magnetômetro "fluxgatè" para satélites científicos. Dissertação (Mestrado em Dispositivos e Sistemas Eletrônicos) - ITA, São José dos Campos; 153 pp.

KABATA W, VITORELLO I, PADILHA AL \& CUNHA WC. 2003. On the dependence of even harmonics with excitation currents in "fluxgate" magnetometers. $8^{\text {th }}$ Int. Cong. Braz. Geophys. Soc., Rio de Janeiro, 4 pp.

KABATA W, VITORELLO I, PÁDUA MB, TRIVEDI NB \& CUNHA WC. 2004.
Magnetômetros fluxgates amorfos robustos para pesquisa em Sondagens Geomagnéticas Profundas no Brasil. Revista de Física Aplicada e Instrumentação, 17(3): 99-109.

PRIMDAHL F. 1970. Temperature Compensation of fluxgate Magnetometers. IEEE Transactions on Magnetics, v. mag6, n. 4. p. 819-822.

RIPKA P. 2003. Switching-Mode fluxgate. The $12^{\text {th }}$ International Conference on Solid-State Sensors, Actuators and Microsystems, Boston, June 8-12.

TANG SC, DUFFY MC, RIPKA P \& HURLEY WG. 2004. Excitation circuit for fluxgate sensor using saturable inductor. Sensors and Actuators A: Physical, 113(2): 156-165.

TRIVEDI NB, OGURA FXK, ANDRADE JC, COSTA JM \& BARRETO LM. 1995. A ring core fluxgate Magnetometer for IEEY Program in Brazil, Revista Brasileira de Geofísica, 13(1): 37-43.

TRIVEDI NB, RUSSEL CT, BARBOSA MJF, DUTRA SLG, TEIXEIRA NR, MEANS JD \& NERI JA. 2000. Geomagnetic field investigation on the polar microsatellite SACI-1. Adv. Space Res., 25(7/8): 1315-1323.

\section{NOTAS SOBRE OS AUTORES}

Wanderlí Kabata. Possui graduação em Engenharia Elétrica pela Universidade Federal de Itajubá (1985), mestrado em Engenharia Eletrônica e Computação pelo Instituto Tecnológico de Aeronáutica (2000) e doutorado em Engenharia Eletrônica e Computação pelo Instituto Tecnológico de Aeronáutica (2006). Atualmente é tecnologista sênior III do Instituto Nacional de Pesquisas Espaciais. Tem experiência na área de Engenharia Elétrica, com ênfase em Instrumentação Eletrônica, atuando principalmente no desenvolvimento de sensores fluxgates de baixo ruído, sensores de efeito Hall para medidas de correntes geomagneticamente induzidas em neutro de transformadores e sistemas autônomos de aquisição de dados de alta resolução e baixo ruído.

Ícaro Vitorello. Atua no INPE em pesquisas e desenvolvimento tecnológico na área de Geomagnetismo, com ênfase em Indução Eletromagnética (Métodos Magnetotelúricos e Sondagens Geomagnéticas Profundas), e em atividades acadêmicas do Curso de Pós-Graduação em Geofísica Espacial. Estudou na Universidade de Michigan (EUA) onde se graduou em Geologia (1972) e realizou a sua pós-graduação em Geofísica da Terra Sólida (Mestrado em Paleomagnetismo - 1975; Doutorado em Estado Térmico da Terra - 1978; Pós-doutorado - 1979). Tem mais de 50 artigos publicados em periódicos arbitrados e mais de cem contribuições em reuniões científicas. Orientou mais de 20 alunos de pós-graduação e inúmeros de iniciação científica. É enquadrado como Pesquisador Titular no INPE e nível I-A no CNPq. Tem participado ativamente em associações científicas (SBGf, AGU, SBG, SBPC), na promoção de reuniões científicas, em atividades editoriais (RBGf e RBG), bancas examinadoras e concursos públicos e contribuído como assessor em diversos programas de fomento à pesquisa (CNPq, FAPESP, PADCT). É sócio honorário da SBGf, tendo exercido diversas funções desde a sua filiação em 1980. 